\title{
Radiological-pathological signatures of patients with COVID-19- related pneumomediastinum: is there a role for the Sonic hedgehog and Wnt5a pathways?
}

\begin{abstract}
To the Editor:
Copyright @The authors 2021

This version is distributed under the terms of the Creative Commons Attribution NonCommercial Licence 4.0. For commercial reproduction rights and permissions contact permissions@ersnet.org

Received: 21 May 2021 Accepted: 14 June 2021

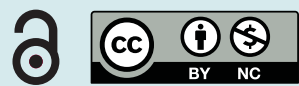

Pneumomediastinum is a rare complication of acute respiratory distress syndrome (ARDS), when air leaks into the mediastinum. An increased pneumomediastinum incidence, of up to 5-13\%, was reported during the coronavirus disease 2019 (COVID-19) pandemic [1, 2] and even occurred spontaneously without a history of mechanical ventilation [3], similarly to the previous severe acute respiratory syndrome (SARS)-1 [4]. Almost half of the 30 consecutive COVID-19 patients who had prolonged invasive mechanical ventilation had full-thickness tracheal lesions and pneumomediastinum [5]. Although pronation and high positive end-expiratory pressure levels were presumed to be the putative causes of pneumomediastinum [5], the mechanism of this COVID-19 complication remains unknown. We hypothesise that sonic hedgehog (SHH) and Wnt5a signalling, crucial pathways in tracheal morphogenesis, and repair/regeneration of cartilage lesions in adulthood [6, 7], could play a role in pneumomediastinum-related COVID-19 tracheal lesions.

This study assessed the pneumomediastinum characteristics in hospitalised COVID-19 patients through a retrospective analysis of a prospectively collected COVID-19 database to identify the clinical-radiological and pathological signatures associated with severe SARS-coronavirus-2 viral infection.

This observational study was carried out from 10 March 2020 to 28 February 2021 at the University Hospital of Trieste and was approved by the local Ethical Committee (CEUR\#2020-Os-148). The patient or next of kin provided written informed consent.

Inclusion criteria were: 1) hospitalised patients with pneumonia positive for COVID-19 confirmed by a positive reverse-transcription-PCR analysis from nasopharyngeal or oropharyngeal swabs [8]; 2) aged $\geqslant 18$ years; 3) chest computed tomography (CT) scan confirmed pneumomediastinum. Volumetric unenhanced CT images were acquired (Revolution EVO GE Healthcare) and reconstructed with a slice thickness of $1 \mathrm{~mm}$ and lung sharp kernels. All CT images were evaluated by three radiologists aware of the COVID-19 status, but blinded to other clinical information. Clinical data were obtained from the local electronic clinical records. Three pathologists blinded to patient identification performed a masked pathological study on the autoptic trachea specimens from patients who died of pneumomediastinum. The patients' haematoxylin and eosin slides were reviewed separately and anonymously. Immunohistochemistry (IHC) was performed on formalin-fixed and paraffin-embedded (4-Gm-thick) sections using antibodies directed against SHH (Abcam; clone EP1190Y, rabbit monoclonal anti-human, 1:100) and Wnt5a (ThermoFisher; clone 3D10, mouse anti-human monoclonal, 1:200). IHC assays were assessed by a semi-quantitative ranking score, ranging from 0 , for no labelling, to 4 for intense labelling, in the slices of cartilage ring remodelling [9].

Statistical analysis were performed using the software $\mathrm{R}$ (rel. 4.0.2). The Gaussian distribution of continuous variables was assessed by the Shapiro-Wilk test. The study population characteristics were assessed by mean \pm SD or median (interquartile range), as appropriate. Continuous variables were compared by t-tests or Mann-Whitney tests.

\footnotetext{
Shareable abstract (@ERSpublications)

Pneumomediastinum is a rare complication of ARDS but is more common during \#COVID19. The fibrous hyaline degeneration of the tracheal rings seen in this autoptic series is an original observation that has not been previously described in COVID-19 patients. https://bit.ly/3vxTQde
}

Cite this article as: Baratella E, Bussani R, Zanconati F, et al. Radiological-pathological signatures of patients with COVID-19-related pneumomediastinum: is there a role for the Sonic hedgehog and Wnt5a pathways? ERJ Open Res 2021; 7: 00346-2021 [DOI: 10.1183/23120541.00346-2021].
\end{abstract}


A total of 1098 consecutive patients with COVID-19 pneumonia were reviewed and 54 (4.9\%) out of 1098 had an unenhanced CT scan of the thorax and met the eligibility criteria. There was a $44.6 \%$ pneumomediastinum-associated mortality rate, making it a severe complication of the disease, whilst the study population mortality rate of hospitalised patients was $22.8 \%$ ( $\mathrm{p}$-value=0.002).

The median (interquartile range) age of patients with pneumomediastinum was 73 (64-77) years, 40 (74.1\%) were males $(29.2 \%$ smokers), and most patients $(n=43,81.1 \%)$ had comorbidities (cardiopathy $52.8 \%$, hypertension $50.9 \%$, obesity $31.4 \%$, diabetes $30.2 \%$, cancer $17.3 \%$, COPD $16.6 \%$, immune depression 3.2\%). Pneumomediastinum alone was detected in 40 (74\%) patients, while the remaining 14 patients had associated pneumothorax (10 mono-lateral partial, three mono-lateral complete, one bi-lateral pneumothorax). The prevalent lung parenchyma CT scan pattern was ground glass in 20 (37\%) patients, alveolar filling in 11 (20.4\%) patients, mixed in 10 (18.5\%) patients, crazy paving in seven (13\%) patients, and reticular/fibrotic pattern in six (11.1\%) patients. Spontaneous pneumomediastinum occurred in four $(7.4 \%)$ patients, one $(1.8 \%)$ patient received high-flow nasal cannula while they had Pneumomediastinum, 30 (55.6\%) patients received noninvasive ventilation/continuous positive airway pressure, and 19 (35.2\%) patients were invasively ventilated.

We observed no statistically significant differences regarding clinical characteristics, co-morbidity, and CT scan features between deceased and surviving patients. Only invasive mechanical ventilation was significantly associated with death.

There was a 9 day median duration (IQR 3-13 day) of mechanical ventilation from intubation to pneumomediastinum. An autopsy was carried out on the 23 patients with pneumomediastinum who died found all of them had tracheal/large airway lesions. A control group of eight patients who died of non-COVID-19-ARDS were also pathologically studied. Autopsy showed that most of the COVID-19 pneumomediastinum patients had full thickness tracheal/large airway lesions (29 (53.7\%) patients) and two had tracheal fistula (figure 1a), while the remaining 16 pneumomediastinum patients had tracheal mucosal ulcers and/or cartilage lesions. All the autopsies showed peculiar findings of the tracheal/airways cartilage, specifically fibrous-hyaline degeneration (figure $1 \mathrm{~b}$ and $\mathrm{c}$ ), that were not present in the trachea and main bronchi of control ARDS subjects (figure 1d). The IHC staining evidenced a strong Wnt5a expression
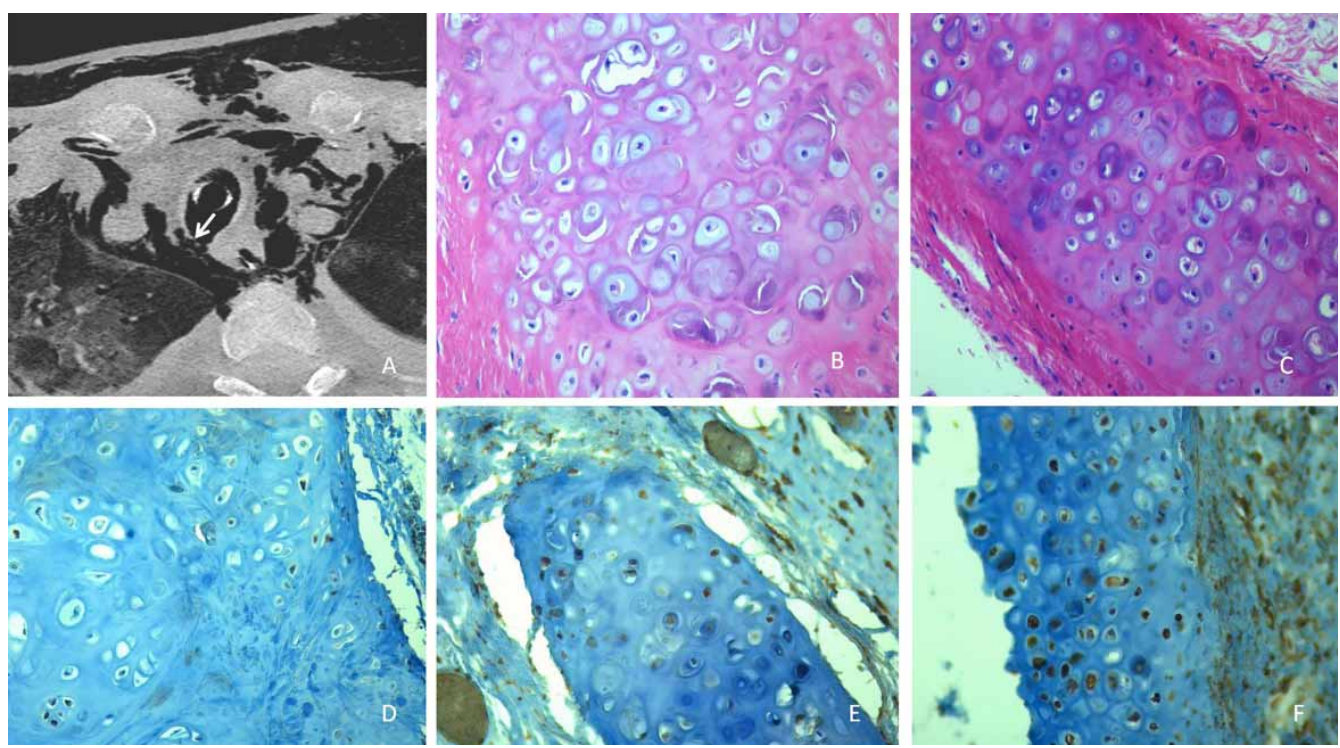

FIGURE 1 a) Axial chest computed tomography scan showing a full-thickness tracheal lesion with fistula. b) Tracheal cartilage micro-cracks in a patient with coronavirus disease 2019 (COVID-19) and pneumomediastinum (Haematoxylin and eosin $\times 40$ ). c) Basophilic cartilage matrix and intracellular fracture with chondrocyte apoptosis (Haematoxylin and eosin $\times 20$ ). d) Control non-COVID-acute respiratory distress syndrome with absent Sonic Hedgehog (Immunohistochemistry $\times 20$ ). e) Weak positive Sonic Hedgehog tracheal chondrocytes in a COVID-19 patient (Immunohistochemistry $\times 20$ ). f) Strong expression of Wnt5a in the same patient (Immunohistochemistry $\times 20)$. 
(labelling score 3.69 \pm 0.55 ) and a weak SHH expression (labelling score $2.21 \pm 0.90$ ) in the cartilage cells of the tracheal/bronchial rings of the COVID-19 patients with pneumomediastinum (figure 1e). Controls were negative for both Wnt5a and SHH for cartilage regeneration biomarkers (labelling score $0.25 \pm 0.46$ for both SHH and Wnt5a, p=0.0001).

Both spontaneous and mechanical ventilation-related pneumomediastinum was observed in our series of COVID-19 patients. Moreover, pneumomediastinum was more isolated than associated to pneumothorax. Autopsies of patients with pneumomediastinum evidenced diffuse tracheal/large airway cartilage lesions with fibrous-hyaline degeneration. Although some of these lesions could represent an iatrogenic effect of mechanical ventilation and a superimposed bacterial infection, no association with intubation or bacterial pneumonia was observed. Therefore, they more likely represent an intrinsic feature of COVID-19.

Other authors already observed an increased pneumomediastinum incidence which was not explainable by barotrauma or high transpulmonary pressure. Our findings are consistent with cartilage remodelling during COVID-19 and ARDS, without a complete post-injury regenerative process [10]. Indeed, two major pathways of chrondrocyte regeneration had a significantly different expression in our autoptic samples, i.e. aberrantly expressed Wnt5a and weakly expressed SHH in the injured cartilage tissue. This is quite different to the usual molecular crosstalk involving Wnt5a and SHH during tracheal development and adult tissue regeneration [7]. In our pneumomediastinum patients tracheal and large airways were frequently injured and an abnormal regenerative process with cartilage tissue remodelling occurred as a pathologic signature, absent in non-COVID-ARDS controls. The fibrous-hyaline degeneration of the tracheal rings seen in our autoptic series is an original observation which has not been previously described in COVID-19 patients.

\section{Elisa Baratella $\oplus^{1}$, Rossana Bussani ${ }^{2}$, Fabrizio Zanconati ${ }^{2}$, Cristina Marrocchio ${ }^{1}$, Giudici Fabiola ${ }^{3,4}$, Luca Braga ${ }^{5}$, Serena Maiocchi ${ }^{5}$, Giorgio Berlot ${ }^{6}$, Maria Concetta Volpe $\oplus^{5}$, Edoardo Moro ${ }^{6}$, Paola Confalonieri ${ }^{7}$, Maria Assunta Cova ${ }^{1}$, Marco Confalonieri ${ }^{7}$, Francesco Salton ${ }^{7}$ and Barbara Ruaro (i) ${ }^{7}$}

${ }^{1}$ Dept of Radiology, Cattinara Hospital, University of Trieste, Trieste, Italy. ${ }^{2}$ Dept of Pathology, Cattinara University Hospital, Trieste, Italy. ${ }^{3}$ Biostatistics Unit, Dept of Medicine, Surgery and Health Sciences, Cattinara Hospital, University of Trieste, Trieste, Italy. ${ }^{4}$ Unit of Biostatistics, Epidemiology and Public Health, Dept of Cardiac, Thoracic, Vascular Sciences and Public Health, University of Padua, Padua, Italy. ${ }^{5}$ Functional Cell Biology Unit, ICGEB, Trieste, Italy. ${ }^{6}$ Intensive Care Unit, Cattinara University Hospital, Trieste, Italy. ${ }^{7}$ Pulmonology Dept, Cattinara University Hospital, Trieste, Italy.

Corresponding author: Barbara Ruaro (barbara.ruaro@yahoo.it)

Acknowledgements: The authors would like to thank Barbara Wade, contract professor at the University of Turin, for her linguistic advice.

Conflict of interest: None declared.

Submitted article, peer reviewed.

References

$1 \quad$ Mart MF, Norfolk SG, Flemmons LN, et al. Pneumomediastinum in acute respiratory distress syndrome from COVID-19. Am J Respir Crit Care Med 2021; 203: 237-238.

2 Lemmers DHL, Hilal MA, Bnà C, et al. Pneumomediastinum and subcutanneous emphysema in COVID-19: barotrauma or lung frailty? ERJ Open Res 2020; 6: 00385-2020.

3 Diaz A, Patel D, Sayedy N, et al. COVID-19 and spontaneous pneumomediastinum: a case series. Heart Lung 2021; 50: 202-205.

4 Chu CM, Leung YY, Hui JYH, et al. Spontaneous pneumomediastinum in patients with severe acute respiratory syndrome. Eur Respir J 2004; 23: 802-804.

5 Fiacchini G, Tric D, Ribechini A, et al. Evaluation of the incidence and potential mechanisms of tracheal complications in patients with COVID-19. JAMA Otolaryngol Head Neck Surg 2021; 147: 70-76.

6 Frank DB, Morrisey EE. Hedgehog and Wnt signaling hubs in tracheal morphogenesis. Am J Respir Crit Care Med 2019; 200: 1202-1204. 
7 Kiyokawa $\mathrm{H}$, Morimoto $\mathrm{M}$. Molecular crosstalk in tracheal development and its recurrence in adult tissue regeneration. Dev Dyn 2021; in press [https://doi.org/10.1002/dvdy.345].

8 Geri P, Salton F, Zuccatosta L, et al. Limited role for bronchoalveolar lavage to exclude COVID-19 after negative upper respiratory tract swabs: a multicentre study. Eur Respir J 2020; 56: 2001733.

9 Fedchenko N, Reifenrath J. Different approaches for interpretation and reporting of immunohistochemistry analysis results in the bone tissue - a review. Diagn Pathol 2014; 9: 221.

10 Nasr T, Holderbaum AM, Chaturvedi P, et al. Disruption of a Hedgehog-Foxf1-Rspo2 signaling axis leads to tracheomalacia and loss of Sox9+ tracheal chondrocytes. Dis Mod Mech 2021; 14: dmm046573. 